J. Clin. Chem. Clin. Biochem.

Vol. 15, 1977, pp. 81-87

\title{
Bestimmung der Urinmetaboliten Hydroxyindolessigsäure, Vanillinmandelsäure und Homovanillin- säure mit lipophiler Gelchromatographie und Gaschromatographie
}

\author{
Von H.-U. Melchert und H. Hoffmeister \\ Aus dem Institut für Sozialmedizin und Epidemiologie des Bundesgesundheitsamtes, Berlin-Dahlem
}

(Eingegangen am 30. Juni/12. Oktober 1976)

Zusammenfassung: Es wird eine spezifische und praktikable Methode zur quantitativen Bestimmung von Phenol-und Indolcarbonsäuren im Harn beschrieben. Die hohe Spezifität wird durch eine Vortrennung der aus den Harnproben extrahierten freien Säuren mit Hilfe der organophilen Gelchromatographie an Sephadex LH 20 und anschließender gaschromatographischer Analyse der Silylderivate der Säuren erreicht. Die organophile Gelchromatographie der freien Säuren zeichnet sich durch hohe Wiederfindungsraten im Mikro- und Submikrogrammbereich aus. Die von anderen Techniken bekannten Schwierigkeiten bei der Derivatisierung und gaschromatographischen Trennung der einzelnen Komponenten werden durch diese Vortrennung und Verwendung von N-Methyl-N-trimethylsilyl-trifluoracetamid als Derivatisierungsmittel überwunden.

Durch diese Aufbereitungstechnik und den Einsatz eines rechnergesteuerten Gaschromatographen mit automatischer Probeneingabe wird eine hohe Richtigkeit und Präzision erreicht und die sichere Bestimmung einer Vielzahl aromatischer Säuren im Harn ermöglicht.

Determination of the urinary metabolites hydroxyindole-acetic acid, vanillyl mandelic acid and homovanillic acid by means of lipophilic gel chromatography and gas chromatography

Summary: A specific and practicable method is described for the quantitative determination of urinary phenoland indole-carboxylic acids. High specificity is achieved by a preliminary separation of the free acids (extracted from the urine sample) with the aid of organophilic gel chromatography on Sephadex LH 20, followed by gas chromatographic analysis of the silyl derivatives of the acids. The organophilic gel chromatography of the free acids shows a high recovery rate in the micro- and submicrogram range. The difficulties encountered in other techniques in the derivatisation and gas chromatographic separation of the individual components are avoided by using the preliminary separation, and by using $\mathrm{N}$-methyl-N-trimethylsilyl-trifluoroacetamide for the derivatisation.

Use of this preparation technique with a direct read-out gas chromatograph with automatic sample introduction, gives high accuracy and precision, and a facility for the determination of a wide range of aromatic acids in urine.

\section{Einführung}

Die qualitative und quantitative Bestimmung von aromatischen Säuren im Harn ist bei der Diagnostik von catecholaminproduzzierenden Tumoren, endokrin aktiven Carcinoiden oder Aminosäure-Stoffwechselanomalien von großer Bedeutung. Bisher wurden in der Analytik dieser Substanzen die Spektralphotometrie, Dünnschichtund Gaschromatographie șowie die Massenspektrometrie bzw. -fragmentographie eingesetzt. $H$. Wisser \& $E$. Knoll (1) veröffentlichten 1973 eine gute Übersicht über die verschiedenen Bestimmungsverfahren. Wie aus verschiedenen Untersuchungen (2) bekannt ist, wird die quantitative Bestimmung der Vanillinmandelsäure nach der Technik von Pisano (3) durch eine Anzahl von gängigen
Arzneimitteln oder deren Metaboliten sowie körpereigene Stoffwechselprodukte gestört. Durch diese Störsubstanzen kann sowohl eine zu geringe, als auch eine zu hohe Ausscheidung von Vanillinmandelsäure vorgetäuscht werden. Ähnliche Probleme bestehen bei der Bestimmung anderer Säuren des Harns. Die Dünnschichtchromatographie wird wegen der Instabilität der zu untersuchenden Substanzen auf der Dünnschichtchromatographie-Platte und anderer Störeinflüsse vorwiegend als halbquantitative Bestimmung durchgeführt. Der $L a$ Brosse-Schnelltest zur Erkennung von Phäochromocytomen und Neuroblastomen ist nach Untersuchungen von $R$. E. Johnsonbaugh et al. (4) nicht empfindlich genug und birgt die Gefahr falsch-negativer Aussagen. 
Die Gaschromatographie bzw. GaschromatographieMassenspektrometrie-Koppelung bietet heute das wohl zuverlässigste Instrument sowohl zur qualitativen als auch zur quantitativen Bestimmung der Säuren im Harn. Die bisher beschriebenen Methoden benötigten jedoch in fast allen Fällen zur Überführung der nicht flüchtigen Säuren in für die Chromatographie geeignete Derivate mehrere Derivatisierungsschritte, bei deren Ausführung die Bildung unerwünschter Nebenprodukte nicht immer sicher auszuschließen ist. So kommt es bei der Methylierung mit Diazomethan leicht zur Bildung von Methyläthern und bei der Derivatisierung mit Trifluoressigsäureanhydrid kann nach Wilk et al. (5) auch mit Nebenreaktionen gerechnet werden, wenn die Derivatisierung des durch Perjodatoxidation aus Vanillinmandelsäure gebildeten Vanillins vorgenommen wird.

Diese Schwierigkeiten und der für Routinezwecke ungünstige Einsatz des giftigen und explosiblen Diazomethans führte uns dazu, die Gaschromatographie der Silylderivate durchzuführen. Für die Silylierung erwies sich das von $M$. Donike (6) beschriebene NMethyl-N-trimethylsilyltrifluoracetamid als besonders geeignet, da es, bedingt durch die hohe eigene Flüchtigkeit und die Flüchtigkeit des bei der Reaktion entstehenden N-Methyl-trifluoracetamids, auch eine Bestimmung der niedermolekularen Substanzen mit verhältnismäßig kurzer Retentionszeit zuläßt. Die Gaschromatographie einer Vielzahl von Säuren als Trimethylsilyl-Derivate wurde bereits 1966 von C. E. Dalgliesh et al. (7), 1968 von W. Ruge (8) sowie 1969 von R. F. Coward \& P. Smith (9) beschrieben. 1969 wurde von T. J. Sprinkle et al. (10) die Bestimmung von Vanillinmandelsäure und Homovanillinsäure durch Gaschromatographie eines silylierten sauren Urinextraktes beschrieben. Diese Methode ist nach Untersuchungen von A. Lanser et al. (11) nur zur Bestimmung stark erhöhter Vanillinmandelsäure- bzw. Homovanillinsäurespiegel brauchbar und nicht zur Ermittlung normalerweise vorliegender Konzentrationen. Wir können diese Untersuchungen bestätigen. Die bei dieser Technik störende Komponente ist die Hippursäure, die in Mengen bis zu $1 \mathrm{~g} / 24 \mathrm{~h}$ ausgeschieden wird.

Aus diesem Grunde, und um die Möglichkeit zu nutzen, mit einer empfindlichen und spezifischen Methode ein Profil der Säuren des Harns zu erstellen, wurde die organophile Gelchromatographie an Sephadex LH 20 zur Vorfraktionierung der aus dem Harn extrahierten freien Säuren verwendet. Der Einsatz von Sephadex LH 20 hat sich bereits bei der Isolierung von Tocopherolen aus Pflanzenölen (12), Steroiden (13), Linolsäuremethylesterhydroperoxiden (14) sowie von Digoxin und Digoxin-Metaboliten (15) bewährt.

Der 1970 von E. Anggard et al. (16) beschriebene Einsatz von LH 20 zur Gelchromatographie phenolischer Säuren erfordert die Herstellung von Methylestern, in der Regel mit Hilfe von Diazomethan, dessen Nachteile schon erwähnt wurden. Die von uns gewählte Arbeitstechnik ermöglicht die Chromatographie der freien aus dem Harn extrahierten Säuren an Sephadex LH 20 und hat gegenüber anderen Vorreinigungs- und Isolierungstechniken, welche den Einsatz von Ionenaustauschern beschreiben (17), den Vorteil, daß die Substanzen in leicht flüchtigem organischen Lösungsmittel anfallen und nach dem schnellen Abdestillieren des Elutionsmittels sofort derivatisiert werden können.

\section{Material und Methoden \\ Geräte}

Schüttelmaschine W 3, Fa. Hormuth-Vetter, Wièsloch.

Chromatographiesäule Chromatronix-Cheminert Typ LC 1/2-13, Fa. Latek, Heidelberg.

Fraktionssammler Linear II, Fa. Serva, Heidelberg.

Durchflußphotometer Uvicord I. Fa. LKB, Gräfelfing.

Gaschromatograph Typ 5830 mit Probengeber 7661 A, Fa.

Hewlett-Packard, Böblingen.

Die Probenaufgabe erfolgte mit Hamilton-Mikroliterspitzen vom Typ $701 \mathrm{~N}$.

\section{Chemikalien}

$m$-Hydroxyphenylessigsäure, $D, L-3,4$-Dihydroxy mandelsäure, 4-Hydroxy-3-methoxyphenylessigsäure (Homovanillinsäure),

$D, L$-4-Hydroxy-3-methoxymandelsäure (Vanillinmandelsäure), Phenylbrenztraubensäure, $p$ Hydroxyphenylbrenztraubensäure, 2,5-Dihydroxyphenylessigsäure (Homogentisinsäure), Salicylursäure, 3-Indolylessigsäure, $D, L$-3-(3-Indolyl)-milchsäure, 5-Hydroxy-3-indolylessigsäure, alle von EGA-Chemie, Steinheim/Albuch. 3,4-Dihydroxyphenylessigsäure, Carl Roth, Karlsruhe. 3-Phenyl-milchsäure, 3-Hydroxy-4-methoxymandelsäure (iso-Vanillinmandelsäure), Fluka Feinchemikalien $\mathrm{GmbH}$, Neu-Ulm.

Sephadex LH 20, Pharmacia Fine Chemicals AB, Uppsala. N-Methyl-N-trimethylsilyl-trifluoracetamid, OV 101 , OV 17 , Gas-Chrom Q, alle von Macherey-Nagel \& Co., Düren.

UCW 982, Chromosorb WAW DMCS, Serva International, Heidelberg.

Alle anderen Chemikalien waren von der Qualität z. A. und wurden von E. Merck, Darmstadt, bezogen.

\section{Ausführung und Bestimmung}

Die folgende Darstellung zeigt die einzelnen Arbeitsschritte des Analysenganges, deren Ausführung dann im einzelnen beschrieben wird.

\section{Extraktion}

$10 \mathrm{ml}$ eines 24-Stunden-Harns (mit $\mathrm{HCl}$ auf $\mathrm{pH} 1$ eingestellt) werden mit $\mathrm{NaCl}$ gesättigt und unter Verwendung einer Schüttelmaschine zweimal für drei Minuten mit je $20 \mathrm{ml}$ Ethylacetat extrahiert. Die vereinigten. Ethylacetatphasen werden ebenfalls für drei Minuten zweimal mit je $10 \mathrm{ml}$ einer $\mathrm{NaHCO}_{3} / \mathrm{K}_{2} \mathrm{CO}_{3}$ Lösung ( $50 \mathrm{~g} \mathrm{NaHCO}_{3}$ und $50 \mathrm{~g} \mathrm{~K}_{2} \mathrm{CO}_{3}$ in 11 Wasser) extrahiert und die organische Phàse verworfen. Die wäßrige Phase wiṛd mit konz. $\mathrm{HCl}$ auf pH 1 gebracht und $\mathrm{NaCl}$ bis zur Sättigung zugefügt. Dann wird erneut zweimal für drei Minuten mit je $20 \mathrm{ml}$ Ethylacetat extrahiert. Die vereinigten Ethylaçetat. phasen werden mit wasserfreiem $\mathrm{Na}_{2} \mathrm{SO}_{4}$ getrocknet, über Quaržwatte in einen Kolben mit ausgezogener Spitze filtrièrt und bei einer Wasserbadtemperatur von maximal $35^{\circ} \mathrm{C}$ unter Wasserstrahlvakuum abdestilliert. Der verbliebene Rückstand wird mit $1 \mathrm{ml} \mathrm{CHCl} /$ Ethanol (Volumina, $4 \mathrm{ml}+1 \cdot \mathrm{ml}$ ) aufgenommen und $0,5 \mathrm{ml}$ davon zur Gelchromatographie verweñdet. 


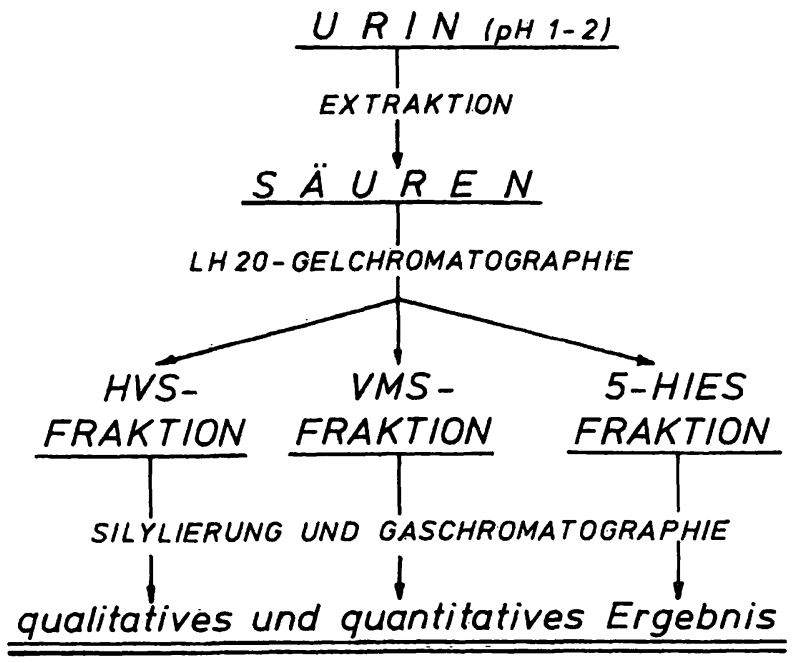

Schema des Analysenverfahrens
Brennluft:

Ofenraum:

Detektor:

Einspritzblock: $250^{\circ} \mathrm{C}$

Als Bezugssubstanzen für die Sicherung der qualitativen Aussage eignen sich die im Urin regelmäßig vorkommende Hippursäure und $p$-Hydroxyphenylessigsäure. Auch Phenylpropionsäure bzw. aliphatische Kohlenwasserstoffe wie z. B. Tetracosan eignen sich als Bezugssubstanzen. Diese können bei der Derivatisierung leicht als Standards zugefügt werden.

Die quantitative Auswertung der Gaschromatogramme erfolgte mit Hilfe von Standardgeraden, welche für die einzelnen Substanzen erstellt wurden.
Die übrige Lösung kann nach Abdestillieren des Lösungsmittels und Silylierung mit $500 \mu \mathrm{l}$ N-Methyl-N-trimethylsilyl-trifluoracetamid zur Erstellung eines qualitativen Übersichtsspektrums der Säuren der Harnprobe verwendet werden.

\section{Gelchromatographie}

Die Gelchromatographie an Sephadex LH 20 wird in einem Chromatographierohr durchgeführt, das mit zwei Durchflußadaptern ausgerüstet ist. Das Gelbett hatte eine Länge von $17,3 \mathrm{~cm}$. Das entspricht einem Gelbettvolumen von $22 \mathrm{ml}$. Die $\mathrm{Zu}$ - und Ableitungen bestanden aus Teflon-Schläuchen von $0,8 \mathrm{~mm}$ Innendurchmesser. Als Elutionsmittel diente ein $\mathrm{CHCl}_{3} / \mathrm{C}_{2} \mathrm{H}_{5} \mathrm{OH}-\mathrm{Gemisch}\left(200 \mathrm{ml} \mathrm{CHCl} 3+50 \mathrm{ml} \mathrm{C}_{2} \mathrm{H}_{5} \mathrm{OH}\right)$. Die Durchflußgeschwindigkeit betrug $30-35 \mathrm{ml} / \mathrm{h}$. Die Elutionsvolumina der einzelnen Substanzen sind von der Fließgeschwindigkeit weitgehend unabhängig. Das Eluat wurde mit einem Fraktionssammler aufgefangen. Zur Ermittlung der Elutionsvolumina wurde die UV-Absorption des Eluats bei $254 \mathrm{~nm}$ kontinuierlich gemessen und als Elutionsdiagramm registriert (Abb. 1). Unter den von uns gewählten Bedingungen zeigte die LH 20-Säule über bisher 12 Monate keine Veränderụng der Trenneigenschaften und der Elutionsvolumina der einzelnen Substanzen.

\section{Derivatisierung und Gaschromatographie}

Die nach vollständigem Abdestillieren des Elutionsmittels verbliebenen Rückstände der LH 20-Fraktionen werden mit $250 \mu \mathrm{l} \mathrm{N}$-Methyl-N-trimethylsilyl-trifluoracetamid versetzt und 30 Minuten auf $60^{\circ} \mathrm{C}$ erhitzt. Die entstehenden Silylderivate sind bei Feuchtigkeitsausschluß für mindestens eine Woche stabil.

Beim Einsatz dieses Derivatisierungsmittels treten Ablagerungen von $\mathrm{SiO}_{2}$ im Flammenionisationsdetektor nach einem Dauerbetrieb von 14 Tagen nur ịn geringer Menge auf. Die Derivate eignen sich gut zum Einsatz im automatischen Probengeber. Die Gaschromatogrạphie wurde unter folgenden Bedingungen durchgeführt.

Säulen:

1. Stahl, $180 \mathrm{~cm}$ lang, $1 / 8^{\prime \prime} \phi, 3 \%$ ov 17 auf Gas-Chrom Q 100-120 mesh

2. Stáhl, $50 \mathrm{~cm}$ lang, $1 / 8^{\prime \prime} \phi, 3 \%$ OV 101 auf Gas-Chrom Q 100-120 mesh

3. Stahl, $180 \mathrm{~cm}$ lang, $1 / 8^{\prime \prime} \phi, 10 \%$ UCW 982 auf Chrómosorb W AW DMCS 80-100 mesh

Trägergas: $\quad$ Säule 1: $35 \mathrm{ml} / \mathrm{min} \mathrm{N}_{2}$

Șäule 2: $20 \mathrm{ml} / \mathrm{min} \mathrm{N}_{2}$

Säule 3: $36 \mathrm{ml} / \mathrm{min} \mathbf{N}_{2}$

Brenngas: $\quad 35 \mathrm{ml} / \mathrm{min} \mathrm{N}_{2}$

\section{Ergebnisse}

Die Abbildung 1 zeigt die Elutionsdiagramme eines Gemisches von Testsubstanzen und eines Urinextraktes sowie die Schnittstellen für die Gewinnung der einzelnen Fraktionen (siehe Schema zur Ausführung der Bestimmung). Die mit Hilfe der Testverbindungen einmal ermittelten Elutionsvolumina blieben über den Zeitraum eines Jahres konstant, so daß die Verwendung eines Fraktionssammlers nach Festlegung der Elutionsvolumina entbehrlich ist. Die aufgefangenen Fraktionen werden bei einer Wasserbadtemperatur von maximal $35^{\circ} \mathrm{C}$ unter Wasserstrahlvakuum vollständig vom Elutionsmittel befreit. Der im Spitzkolben verbliebene Rückstand wird dann zur Silylierung und anschließenden Gaschromatographie verwendet.

Die Abbildung 2 zeigt das Gaschromatogramm einer Anzahl von Testverbindungen auf der OV 17-Säule.

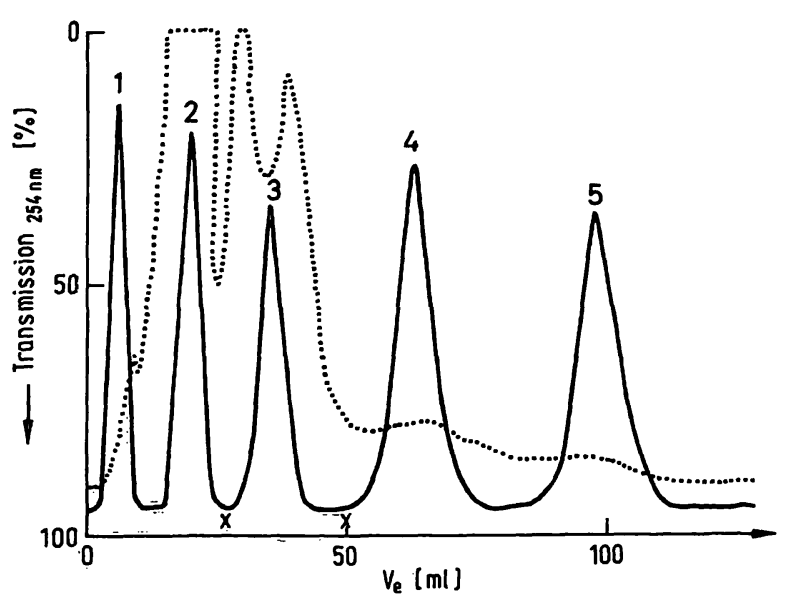

Abb. 1. Elutionsdiagramme eines Gemisches von je $500 \mu \mathrm{g}$ Carotin (1), Homovanillinsäure (2), Vanillinmandelsäure (3), 5-Hydroxyindolessigsäure (4), 3,4-Dihydroxymandelsäure (5) (-) und eines Urinextraktes (....) $\mathrm{X}=$ Fraktionsschnittstellen. 


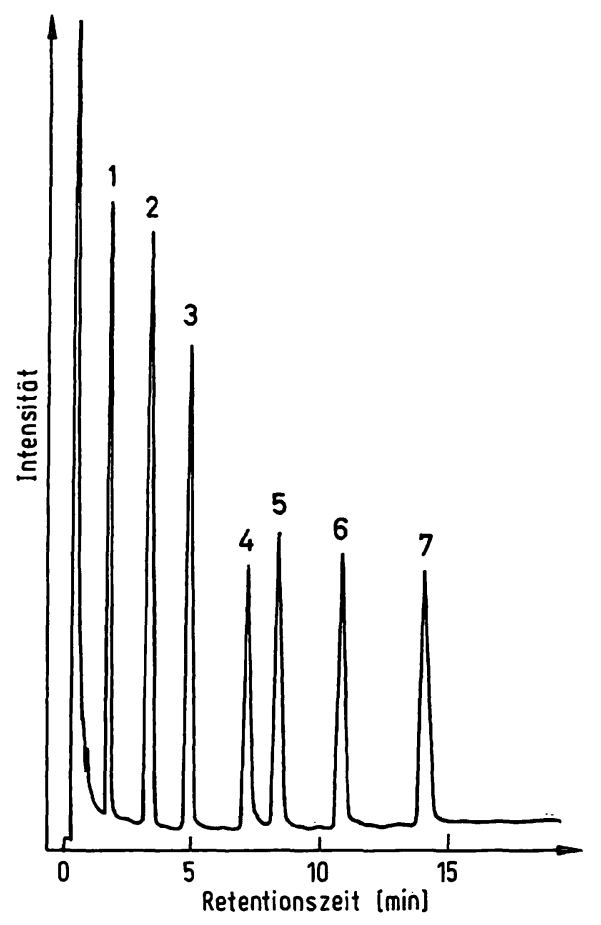

Abb. 2. Gaschromatogramm eines Testgemisches von je $250 \mathrm{ng}$ der Trimethylsilylderivate von Phenylessigsäure (1), Salicylsäure (2), p-Hydroxyphenylessigsäure (3), Homovanillinsäure (4), Vanillinmandelsäure (5), Indolylessigsäure (6) und 5-Hydroxyindolessigsäure (7) auf der OV 17-Säule.

Die Tabelle 1 zeigt, nach Elutionsvolumina geordnet, die Elutionsvolumina und die Retentionszeiten von Testverbindungen.

Zur Gaschromatographie der HomovanillinsäureFraktion eignen sich unpolare Trennflüssigkeiten, wie z. B. OV 101 oder UCW 982. Die Abbildung 3 zeigt die silylierten Säuren eines Urinextraktes aus einem Kontroll-Urin der Firma Lederle mit erhöhten Werten für Vanillinmandelsäure $(13 \mathrm{mg} / \mathrm{l})$ und 5-Hydroxy-3indolylessigsäure $(21 \mathrm{mg} / \mathrm{l})$ sowie einer normalerweise im Harn nicht vorliegenden Substanz, nämlich die Salicylsäure $(230 \mathrm{mg} / \mathrm{l})$.

Die Abbildung 4 zeigt die aus diesem Kontroll-Urin isolierte Homovanillinsäurefraktion, welche Vanillinmandelsäure und 5-Hydroxy-3-indolylessigsäure nicht mehr enthält. Diese beiden Chromatogramme wurden auf der Säule 3 erhalten. Zur Messung der Vanillinmandelsäurebzw. 5-Hydroxy-3-indolyl-essigsäurefraktion werden besser polarere Trennflüssigkeiten, wie z. B. OV 17 , eingesetzt. So zeigt die Abbildung 5 die silylierte Vanillinmandelsäurefraktion eines durch LH 20-Chromato; graphie vorgetrennten Urinextraktes. Die störenden Hippur- und Salicyl-bzw. Salicylursäure-Peaks sind hier nicht mehr vorhanden.

Die Abbildung 6 schließlich zeigt die silylierte 5-Hydroxy3-indolyl-essigsäurefraktion, welche fast ausschließlich den Peak der 5-Hydroxy-3-indolylessigsäure enthält. Den Erfolg der Vortrennung durch die Gelchromatographie
Tab. 1. Elutionsvolumina und Retentionszeiten von Testverbindungen.

\begin{tabular}{|c|c|c|c|}
\hline Substanz & $\begin{array}{l}\text { Elutions- } \\
\text { volumen } \\
{[\mathrm{ml}]}\end{array}$ & $\begin{array}{l}\text { Retentionszeiten [m } \\
\text { OV } 17 \text { - } \\
\text { Säule }\end{array}$ & $\begin{array}{l}\text { ain] } \\
\text { OV } 101- \\
\text { Säule }\end{array}$ \\
\hline $\begin{array}{l}\text { Phenylbrenz- } \\
\text { traubensäure }\end{array}$ & $9-15$ & 16,62 & 12,72 \\
\hline Phenylessigsäure & $13-21$ & 1,81 & 1,15 \\
\hline Benzoesäure & $14-22$ & 1,37 & 0,89 \\
\hline Hippursäure & $14-24$ & $9,60(7,99)$ & 6,98 \\
\hline Homovanillinsäure & $15-25$ & 7,14 & 6,46 \\
\hline Phenylmilchsäure & $16-25$ & 4,01 & 3,85 \\
\hline Salicylursäure & $17-29$ & $12,21(3,35+9,61)$ & $10,39(3,16)$ \\
\hline Vanillinsäure & $19-30$ & 6,81 & 6,40 \\
\hline Salicylsäure & $19-27$ & 3,35 & 3,16 \\
\hline Mandelsäure & $20-31$ & 3,02 & 2,81 \\
\hline 3-Indolylessigsäure & $21-37$ & 10,84 & 8,82 \\
\hline $\begin{array}{l}\text { 2-Hydroxyphènyl- } \\
\text { essigsäure }\end{array}$ & $25-28$ & 4,03 & 3,79 \\
\hline $\begin{array}{l}\text { 3-Hydroxyphenyl- } \\
\text { essigsäure }\end{array}$ & $25-38$ & 4,27 & 4,52 \\
\hline $\begin{array}{l}\text { 4-Hydroxyphenyl- } \\
\text { essigsäure }\end{array}$ & $29-39$ & 4,91 & 4,68 \\
\hline $\begin{array}{l}\text { Vanillinmandel- } \\
\text { säure }\end{array}$ & $26-45$ & 8,38 & 8,34 \\
\hline Indolylmilchsäure & $29-50$ & 13,25 & 12,20 \\
\hline $\begin{array}{l}\text { iso-Vanillin- } \\
\text { mandelsäure }\end{array}$ & $32-49$ & 8,81 & 8,37 \\
\hline $\begin{array}{l}\text { 3,4-Dihydroxyphe- } \\
\text { nylessigsäure }\end{array}$ & $45-65$ & 7,20 & 7,51 \\
\hline $\begin{array}{l}\text { 5-Hydroxyindol- } \\
\text { essigssäure }\end{array}$ & $51-75$ & 14,09 & 12,57 \\
\hline Homogentisinsäure & $55-76$ & 7,35 & $7,5.3$ \\
\hline $\begin{array}{l}\text { 4-Hydroxyphenyl- } \\
\text { brenztrauben- } \\
\text { säure }\end{array}$ & $56-90$ & 10,30 & 10,60 \\
\hline $\begin{array}{l}\text { 3,4-Dihydroxy- } \\
\text { mandelsäure }\end{array}$ & $85-110$ & 8,36 & 9,30 \\
\hline
\end{tabular}

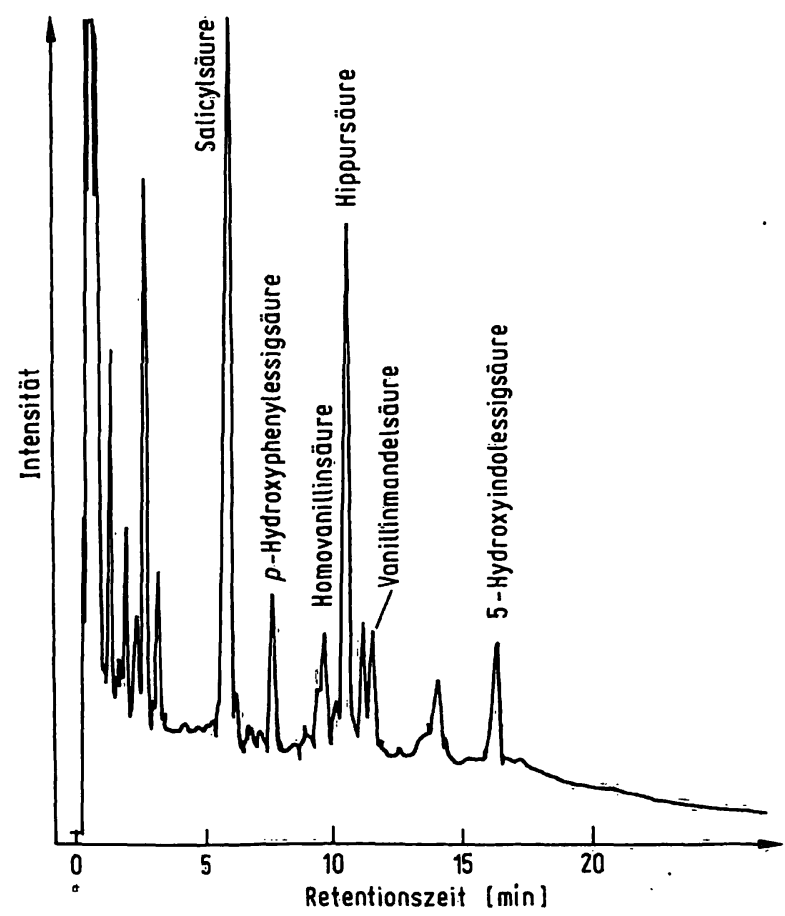

Abb. 3. Gaschromatogramm der silylierteñ Säuren eines Urinextraktes auf der UCW 982-Säule: 


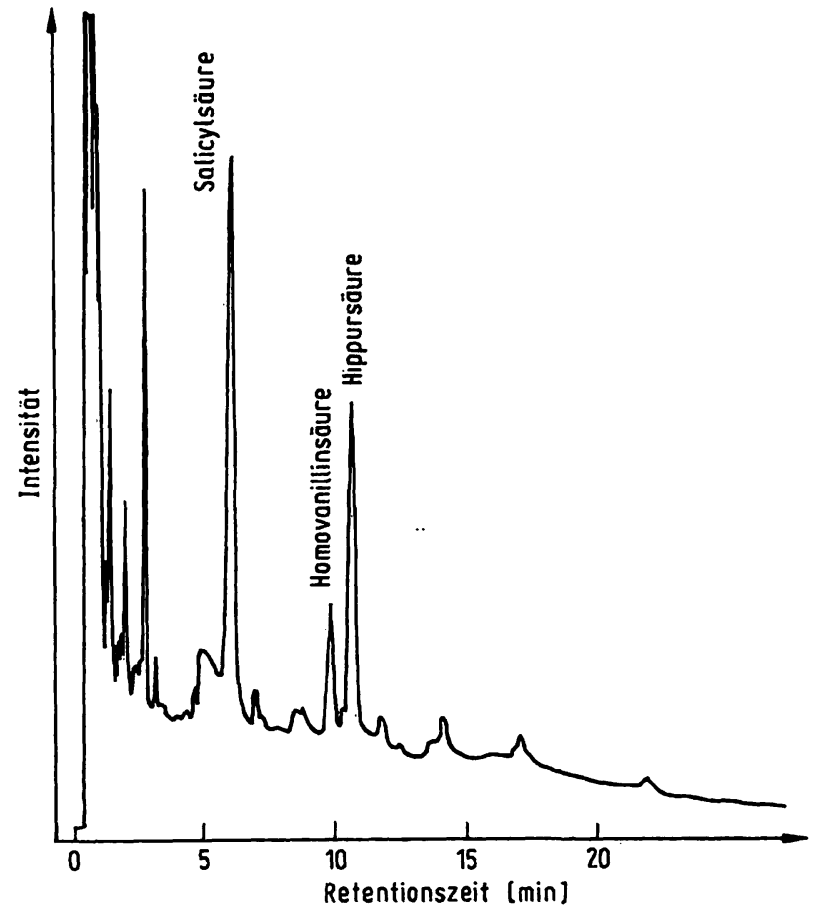

Abb. 4. Gaschromatogramm der silylierten Säuren der Homovanillinsäurefraktion eines Urinextraktes auf der UCW 982-Säule.

zeigt deutlich ein Vergleich der Abbildungen 5 und 6 mit der folgenden Abbildung 7, bei welcher die gesamten durch Extraktion aus dem Harn isolierten Säuren silyliert und auf der OV 17-Säule gaschromatographisch getrennt wurden.

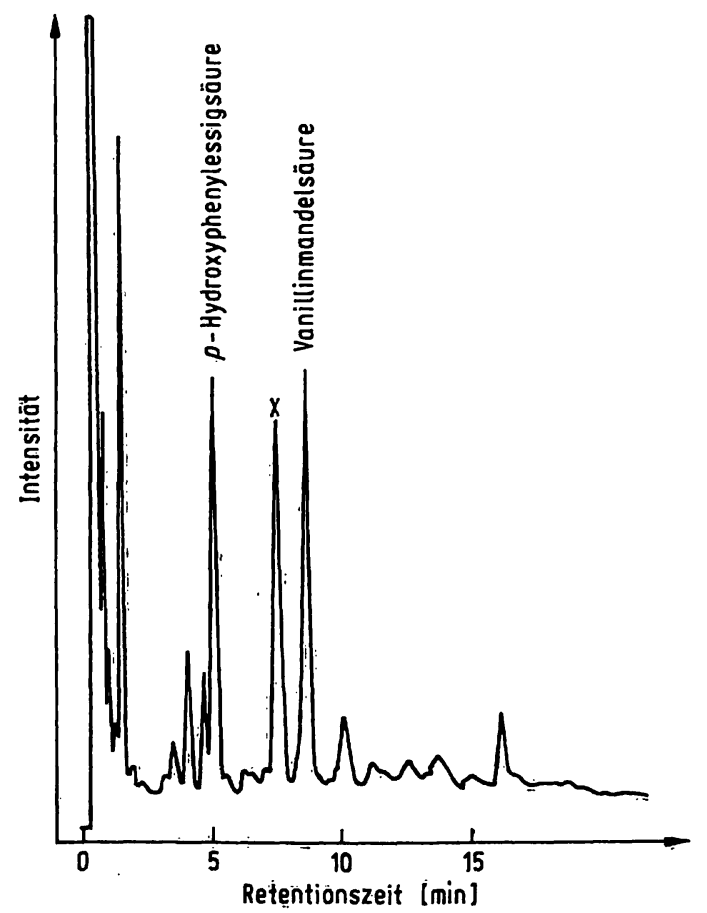

Abb. 5. Gaschromatogramm der silylierten Säuren der Vanillinmandelsäurefraktion eines Ürinextraktes $(X=$ unbekannte Substanz mit ähnlicher Retentionszeit wie Homovanillinsäure, jedoch mit añderem Elutionsvolumen) auf der OV 17-Säule.

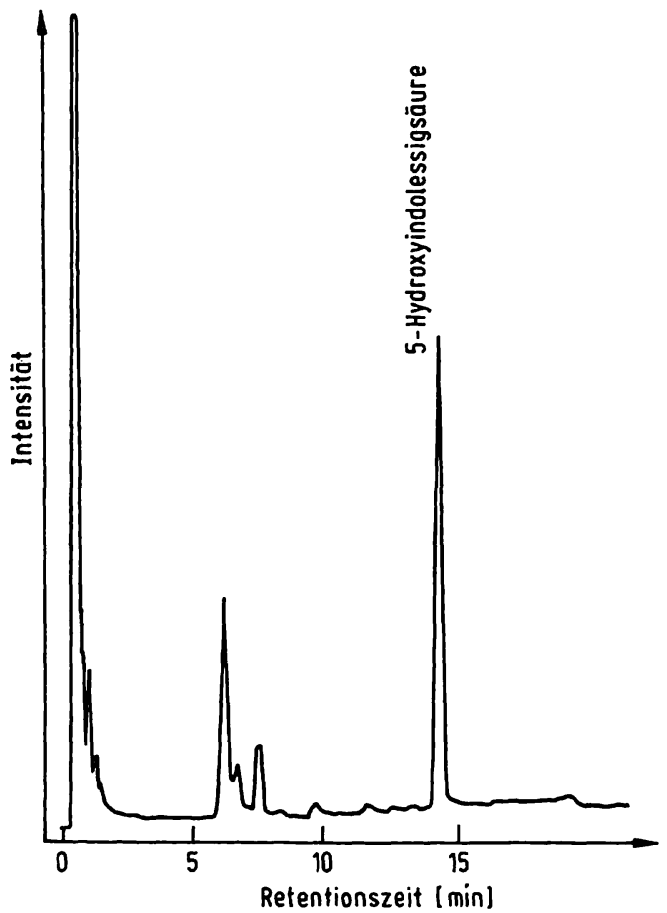

Abb. 6. Gaschromatogramm der silylierten Säuren der 5Hydroxyindolessigsäurefraktion eines Urinextraktes auf der OV 17-Säule.

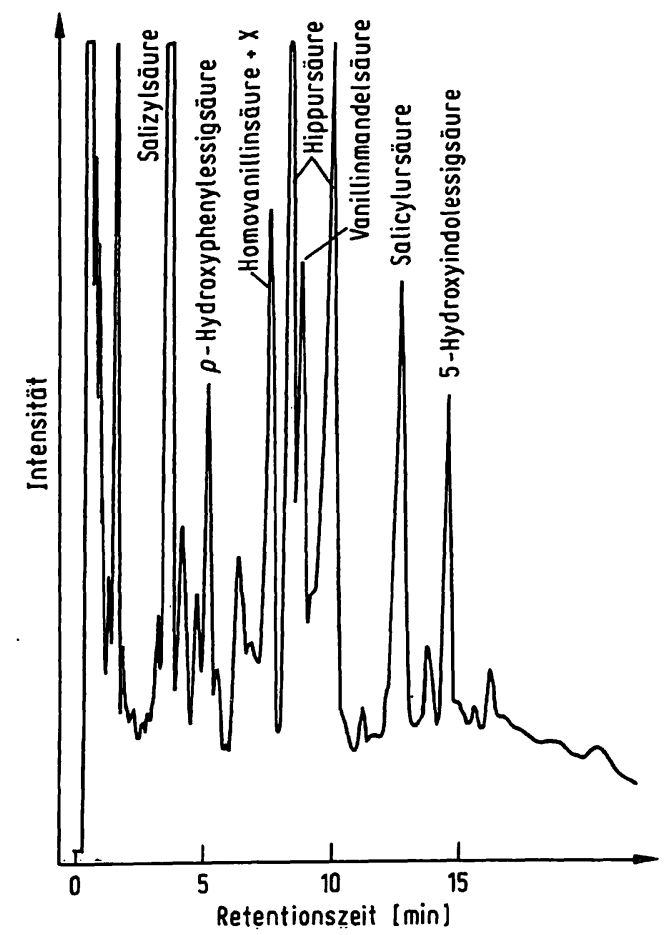

Abb. 7. Gaschromatogramm der silylierten Säuren eines Urinextraktes auf der OV 17-Säule.

\section{Qualitätskriterien}

Zur Kontrolle der Richtigkeit wurden mit Homovanillinsäure, Vanillinmandelsäure und 5-Hydroxy-3-indolylessigsäure Wiederfindungsversuche durchgeführt. Es zeigte sich, daß bei der LH 20-Chromatographie Mengen von 25-100 $\mu \mathrm{g}$ Homovanillinsäure und Vanillinmandelsäure 
als underivatisierte Substanzen mit Wiederfindungsraten von $95-100 \%$ und $25-100 \mu$ g-Hydroxy-3indolylessigsäure mit Wiederfindungsraten von 83-92\% chromatographiert werden können.

Die Wiederfindungsraten für den gesamten Analysengang einschließlich der Extraktion aus dem Harn, bestimmt für zwei verschiedene Substanzmengen, zeigt die folgende Aufstellung:

\begin{tabular}{|c|c|c|}
\hline Substanz & $\begin{array}{l}\text { zugefügte } \\
\text { Mengen }\end{array}$ & $\begin{array}{l}\text { wiedergef undenèr } \\
\text { Anteil }\end{array}$ \\
\hline $\begin{array}{l}\text { Homovanillinsäure } \\
\text { Vanillinmandelsäure } \\
\text { 5-Hydroxy-3- } \\
\text { indolylessigsäure }\end{array}$ & $\begin{array}{l}25 \mu \mathrm{g} \text { bzw. } 100 \mu \mathrm{g} \\
25 \mu \mathrm{g} \mathrm{bzw.} 100 \mu \mathrm{g} \\
25 \mu \mathrm{g} \text { bzw. } 100 \mu \mathrm{g}\end{array}$ & $\begin{array}{l}91 \% \text { bzw. } 95 \% \\
89 \% \text { bzw. } 95 \% \\
80 \% \text { bzw. } 88 \%\end{array}$ \\
\hline
\end{tabular}

Bei der Verwendung von Diethyläther als Extrak- • tionsmittel erhielten wir bei der Wiederfindung von 5-Hydroxy-3-indolyl-essigsäure Wiederfindungsraten von $84 \%$ bzw. $90 \%$.

Die Präzision wurde durch 10 Einzelmessungen an fünf verschiedenen Tagen aus der gleichen Kontroll-Urinprobe ermittelt. Dabei ergaben sich folgende Werte:

\begin{tabular}{lrll}
\hline Substanz & $\bar{x}$ & s & VK \\
\hline Homovanillinsäure & $6,95 \mathrm{mg} / 1$ & $0,32 \mathrm{mg} / 1$ & $4,6 \%$ \\
Vanillinmandelsäure & $9,44 \mathrm{mg} / 1$ & $0,56 \mathrm{mg} / 1$ & $5,9 \%$ \\
5-Hydroxy-3- & $20,25 \mathrm{mg} / 1$ & $1,34 \mathrm{mg} / 1$ & $6,6 \%$ \\
indolylessigsäure & & &
\end{tabular}

\section{Diskussion}

Die wesentlichen Vorteile dieses Bestimmungsverfahrens gegenüber den meisten bisher eingesetzten liegen

- in der hohen Spezifität, gekoppelt mit hoher Empfindlichkeit,

- in einer guten Richtigkeit und Präzision,

- in der Möglichkeit, eine Vielzahl weiterer Säuren qualitativ und quantitativ zu bestimmen, sowie

- in der einfachen und routinemäßigen Durchführbarkeit der Analysen.

Die hohe Spezifität wird durch den Einsatz zwei verschiedener chromatographischer Verfahren erreicht.

\section{Literatur}

1. Wisser, H. \& Knoll, E. (1973), diese Z. 11, 3-14.

2. Féldman, J. M., Butler, S. S. \& Chapman, B. A. (1974), Clin. Chem. 20, 607-610.

3. Pisano, J. J., Crout, J. R. \& Abraham, D. (1962), Clin. Chim. Acta 7, 285-291.

4. Johnsonbaugh, R. E. \& Cahill, R. (1975), Pediatr. 56, (2).

5. Wilk, S., Gitlow, S. E., Mendlowitz, M., Franklin, M. J., Carr, H. E. \& Clarke, D. D. (1965), Anal. Biochem. 13, 544-551.

6. Donike, M. (1969), J. Chromatogr. 42, 103-104.
Wie aus der Zusammenstellung der Elutionsvolumina und Retentionszeiten der Testverbindungen zu ersehen ist, ist die Trennung der aufgeführten Verbindungen weitgehend problemlos. Substanzen, die im gaschromatographischen Trennsystem nur schwer zu trennen sind (z. B. Vanillinmandelsäure und 3,4-Dihydroxymandelsäure auf OV 17 oder Phenylmilchsäure und o-Hydroxyphenylessigsäure auf OV 101) liegen in ihren Elutionsvolumina soweit auseinander, daß durch die Gelchromatographie eine ausreichende Vortrennung möglich ist. Die ungewöhnlich starke Retardierung einiger Substanzen bei der Gelchromatographie ist offenbar durch einen Adsorptionseffekt hervorgerufen, der durch die Anzahl der freien OH-Gruppen bedingt ist, wie er bereits von $W$. Wachs (12) und H.-U. Melchert (18) für Tocopherole und Antioxidantien beschrieben wurde. Richtigkeit und Präzision werden im wesentlichen durch die günstigen Eigenschaften der Gel- und Gaschromatographie bestimmt. Besonders hervorzuheben ist hierbei die Stabilität der Elutionsvolumina sowie der Retentionszeiten. Voraussetzung für dịe reproduzierbare Durchführung der Gelchromatographie ist die Verwendung von Chromatographiesäulen mit zwei Durchflußadaptern, welche die Stabilität des Gelbettes gewährleisten. Das Auswaschen von Substanzen aus dem LH 20-Gel, welche die gaschromatographische Analyse beeinträchtigen würden, konnte nicht beobachtet werden. Die Präzision der gaschromatographischen Analysen wird durch den Einsatz eines automatischen Probengebers wesentlich verbessert.

Diese Art der Analytik bietet die Möglichkeit, eine Vielzahl von sauren, basischen oder neutralen Komponenten - bei geringer Modifizierung der Aufarbeitung der Probe - im Harn qualitativ und quantitativ zu bestimmen.

Daher ist zu erwarten, daß chromatographische Techniken künftig in steigendem Umfang auch für die Routineanalytik zu nutzen sind.

\section{Danksagung}

Frau G. Mathis danken wir für die sorgfältige Durchführung der Analysen.
7. Dalgliesh, C. E., Horning, M. G., Knox, K. L. \& Yarger, K. (1966), Biochem. J. 1.01, 792-810.

8. Runge, W. (1968), diese Z. 6, 448-452.

9. Coward, R. F. \& Smith, P. (1969), J. Chromatogr. 45, 230-243.

10. Spprinkle, T. J., Porter, A. H., Greer, M. \& Williams, C. M. (1969), Clin. Chim. Acta 25, 409-411.

11. Lanser, A., Oldenziel, H., Pronk, C. \& Lequin, H. C. (1974), Clin. Chim. Acta 5., 293-296. 
12. Wachs, W. \& Melchert, H.-U. (1971), Dtsch. Lebensm.Rundsch. 67, 221-225.

13. Lisboa, B. P. \& Strassner, M. (1975), J. Chromatogr. 111, $159-164$.

14. Rubach, K., Schormüller, J. \& Melchert, H.-U. (1971), Lebensm. Ernähr. 9, 166-169.

15. Gault, M. H., Ahmed, M., Symes, A. L. \& Vance, J. (1976), Clin. Biochem. 9, 46-52.
16. Anggard, E., Sjöquist, B. \& Sjöström, R. (1970), J. Chromatogr. 50, 251-259.

17. Thompson, J. A. \& Markey, S. P. (1975), Anal. Chem. 47, 1313-1321.

18. Melchert, H.-U. (1973), Chem. Mikrobiol. Technol. Lebensm. 2, 94-95.
Dr. H.-U. Melchert und Prof. Dr. H. Hoffmeister Institut für Sozialmedizin und Epidemiologie des Bundesgesund heitsamtes Postfach

D-1000 Berlin 33 
